Mens

revue d'histoire intellectuelle de l'Amérique française

\title{
La représentation du régime hitlérien par La Patrie de Montréal (1933-1939)
}

\section{Patrick Poirier}

Volume 4, numéro 1, automne 2003

URI : https://id.erudit.org/iderudit/1024628ar

DOI : https://doi.org/10.7202/1024628ar

Aller au sommaire du numéro

Éditeur(s)

Centre de recherche en civilisation canadienne-française

ISSN

1492-8647 (imprimé)

1927-9299 (numérique)

Découvrir la revue

Citer cet article

Poirier, P. (2003). La représentation du régime hitlérien par La Patrie de Montréal (1933-1939). Mens, 4(1), 69-93. https://doi.org/10.7202/1024628ar

\section{Résumé de l'article}

Les éditorialistes de La Patrie, un quotidien montréalais, adoptent une position généralement tiède à l'égard du régime nazi dans les premiers temps du III ${ }^{\mathrm{e}}$ Reich. Par contre, à partir de 1938, leur position se radicalise, leur discours devient hostile. L'anticatholicisme et le paganisme du régime hitlérien justifient alors la représentation diabolique de Hitler et du régime. Cette prise de position des éditorialistes est influencée, d'une part, par l'intensification des crises internationales et la menace d'une guerre et, d'autre part, par la prise de position officielle du pape Pie XI à partir de 1937. Nous avons repéré, dans le discours des éditorialistes, un amalgame de valeurs traditionnelles et libérales. La vision du monde des éditorialistes de La Patrie nous indique que les valeurs traditionnelles sont encore influentes dans les années 1930 mais qu'elles ont été adaptées par l'intégration de valeurs libérales et de traits caractéristiques de la modernité dans une conception morale de la civilisation.
Ce document est protégé par la loi sur le droit d'auteur. L'utilisation des services d’Érudit (y compris la reproduction) est assujettie à sa politique d'utilisation que vous pouvez consulter en ligne.

https://apropos.erudit.org/fr/usagers/politique-dutilisation/ 


\title{
LA REPRÉSENTATION DU RÉGIME HITLÉRIEN PAR LA PATRIE DE MONTRÉAL (1933-1939)
}

\author{
Patrick Poirier \\ Département d'histoire \\ Université du Québec à Montréal
}

\section{RÉSUMÉ}

Les éditorialistes de La Patrie, un quotidien montréalais, adoptent une position généralement tiède à l'égard du régime nazi dans les premiers temps du III ${ }^{\mathrm{e}}$ Reich. Par contre, à partir de 1938, leur position se radicalise, leur discours devient hostile. L'anticatholicisme et le paganisme du régime hitlérien justifient alors la représentation diabolique de Hitler et du régime. Cette prise de position des éditorialistes est influencée, d'une part, par l'intensification des crises internationales et la menace d'une guerre et, d'autre part, par la prise de position officielle du pape Pie XI à partir de 1937. Nous avons repéré, dans le discours des éditorialistes, un amalgame de valeurs traditionnelles et libérales. La vision du monde des éditorialistes de La Patrie nous indique que les valeurs traditionnelles sont encore influentes dans les années 1930 mais qu'elles ont été adaptées par l'intégration de valeurs libérales et de traits caractéristiques de la modernité dans une conception morale de la civilisation.

\section{ABSTRACT}

The Montreal daily La Patrie adopted a relatively mild stance regarding the Nazi regime in the early days of the Third Reich. However, from 1938 on, Nazipaganism and anti-Catholicism made its editorialists increasingly hostile to Hitler and his regime. This new editorial stance was influenced on the one hand, by the intensification of international tension and the threat of war and, on the other hand, by Pope Pius XI's 1937 condemnation of nazism. Overall, La 
Patrie's editorial stance on the Nazi regime was an amalgamation of liberal and traditional values. This reveals that traditional values remained influential in 1930s Quebec, but that they were being altered by the incorporation of liberal values and modernity into the moral concept of civilisation.

L'étude de la représentation du régime nazi dans le quotidien La Patrie a pour but de comprendre la vision du monde des éditorialistes d'un quotidien à grand tirage à Montréal dans les années 1930. Selon Jacques Pelletier, la notion de vision du monde est essentiellement un outil conceptuel :

La vision du monde est en quelque sorte une attitude première, une prise de position fondamentale à l'endroit de l'univers, de la société et des valeurs, attitude fondamentale qui oriente de façon décisive tous les comportements pratiques de l'homme dans son existence quotidienne, par laquelle s'éclairent ses prises de position sur les problèmes les plus divers qui, à première vue, pourraient sembler discordantes ou totalement incompatibles ${ }^{1}$.

La nature même du régime nazi, son caractère répressif, autoritaire, et ses visées expansionnistes de même que la nature raciste de son idéologie posaient des problèmes d'ordre politique, philosophique et moral à l'observateur de l'époque. La représentation du régime hitlérien et de l'idéologie nazie implique donc une prise de position politique, philosophique et morale. Elle implique un jugement de valeurs, ce jugement étant fondé sur une certaine conception du Bien et du Mal. L'analyse de la représentation de l'Allemagne hitlérienne nous permet de comprendre la vision du monde qui animait le discours et la pensée des observateurs contemporains du régime, en l'occurrence les éditorialistes en tant que commentateurs de l'actualité.

Les politiques intérieures et étrangères de l'État allemand occupent le devant de la scène internationale tout au 
long de la période qui nous intéresse, soit de 1933 à 1939. Nous savons que les événements qui pavent l'instauration de la dictature nazie, dans un premier temps, puis ceux qui marquent la marche vers la guerre, dans un deuxième temps, suscitent l'intérêt de la presse internationale ${ }^{2}$. Ces événements y sont amplement rapportés et commentés. Les quotidiens francophones du Québec suivent cette tendance générale de la presse occidentale. Des études telles que celles de Guy Massicotte $^{3}$ ou de Françoise Caron-Houle ${ }^{4}$ nous ont démontré l'intérêt de la presse d'information d'expression française au Québec pour le régime hitlérien et les événements engendrés par ses actions politiques. Or que savons-nous de l'opinion des journalistes, et plus particulièrement des éditorialistes francophones au Québec sur ces questions? Claude Couture affirme ainsi que « [s]ur le plan politique, le corporatisme, le fascisme, le socialisme n'ont exercé sur cette presse aucune séduction. La presse libérale a sévèrement condamné le nazisme et en particulier, l'antisémitisme ${ }^{5}$. »

Or ce n'est pas suffisant pour expliquer la position des éditorialistes de la presse francophone à grand tirage face au régime hitlérien. Il s'agit aussi de comprendre le discours qui sous-tend cette position. Comme les éditorialistes participent à la culture de leur société, leur vision du monde trouve sa source dans le contexte plus général des idées au Québec dans les années 1930.

La Patrie a été fondé en 1879 par Honoré Beaugrand à la demande de Wilfrid Laurier. Le quotidien était, à cette époque, l'organe officiel du Parti libéral au Québec ; il exprimait alors les tendances radicales du parti et servait de véhicule, selon Hamelin et Beaulieu, au rougisme que Beaugrand cherchait à ressusciter. En 1933, La Presse acquiert La Patrie qui sera alors transformée en profondeur afin d'accroittre son marché. Le quotidien deviendra alors le deuxième parmi les 
journaux francophones les plus lus à Montréal. Pour la période de 1928 à 1939, la moyenne des tirages déclarés par les quotidiens publiés à Montréal était de 152555 exemplaires pour La Presse ; 23 631, pour La Patrie ; 14004 pour Le Devoir; 12986 pour Le Canada ; et, enfin, 8454 pour MontréalMatin ${ }^{7}$.

\section{Les éditorialistes de La Patrie}

De 1933 à 1935, les éditoriaux de La Patrie ne sont pas signés par leur auteur. La signature des éditoriaux n'apparaît qu'aux alentours de 1936. Elle devient pratique courante dans les textes de la période 1938-1939, même si l'on rencontre encore parfois des éditoriaux non signés. Trois personnes signent alors les éditoriaux, Léon Gray (39 éditoriaux), Hervé de Saint-Georges (2 éditoriaux) et Dostaler O'Leary (3 éditoriaux). Nous ne possédons pas d'information sur les deux premiers éditorialistes. Léon Gray semble avoir été à l'époque l'éditorialiste de La Patrie responsable des affaires internationales. Dostaler O'Leary est par contre mieux connu. Ce dernier est né à Berthierville le 16 août 1908 et est décédé à Paris le 18 avril 1965. Il entre à la Patrie en 1937 où il demeure jusqu'en 1957. Il a occupé les postes de rédacteur financier, d'éditorialiste, de directeur de la page littéraire, de chroniqueur de la «vie française » et de courriériste parlementaire à Québec de 1948 à 1956. Il a été membre des JeuneCanada. Il est cofondateur, avec son frère Walter-Patrice, des Jeunesses patriotes du Canada français et des Chevaliers de la table ronde du Canada en 1935, puis de l'Union culturelle Mexique-Canada français en 1939, devenue par la suite l'Union des Latins d'Amérique. Il a été collaborateur de la revue de Paul Bouchard, La Nation et candidat défait du Bloc populaire canadien dans la circonscription de Montréal-Laurier aux élections fédérales de 1945. 


\section{Modernité, tradition et médias à grand tirage}

L'histoire des idées dans la société francophone québécoise est marquée par la question fondamentale de la modernité et de la modernisation de cette société : c'est essentiellement la question de "l'entrée dans la modernité ${ }^{8}$ » et celle du caractère de cette modernité qui posent problème. On s'interroge sur le moment d'une rupture, ou d'une succession de ruptures, qui déterminerait la transformation d'une culture traditionnelle et son passage à une culture moderne. Bien que le concept de modernité ne soit pas universel et qu'il s'applique à un cadre spatio-temporel limité (l'Europe du XIV au $\mathrm{XIX}^{\mathrm{e}}$ siècles ou du $\mathrm{XX}^{\mathrm{e}}$ siècle selon les approches), nous pouvons tirer de sa définition un cadre théorique qui nous permet d'expliquer les changements et les transformations de la société québécoise contemporaine. Pour Jean Baudrillard, «la modernité n'est pas un concept politique, ni proprement un concept historique ${ }^{9} »$ :

C'est un mode de civilisation caractéristique qui s'oppose au mode de la tradition, c'est-à-dire à toutes les autres cultures antérieures ou traditionnelles : face à la diversité géographique et symbolique de celles-ci, la modernité s'impose comme une, homogène, irradiant mondialement à partir de l'Occident. [...] Morale canonique du changement, elle s'oppose à la morale canonique de la tradition, mais elle se garde tout autant du changement radical.[...] Comme elle n'est pas un concept d'analyse, il n'y a pas de lois de la modernité, il n'y a que des traits de la modernité. Il n'y a pas non plus de théorie, mais une logique de la modernité, et une idéologie ${ }^{10}$.

La logique de la modernité peut se définir par trois concepts : le concept techno-scientifique, le concept politique et le concept psychologique. Ces trois concepts, selon 
Baudrillard, ne constituent pas la modernité en soi, mais bien trois niveaux d'intervention de la modernité dans les sociétés. Ils correspondent aux transformations des structures sociales et politiques, aux effets d'un nouveau rapport à la réalité qui a pour fondements la connaissance scientifique, la rationalité et l'individualisme.

Un aspect essentiel de la modernité est son processus. Celui-ci est basé sur le rapport qu'elle entretient avec la tradition. Comme le mentionne Baudrillard, ce processus n'est pas radical :

[...] le terrain de l'anthropologie montre, plus clairement que l'histoire européenne, la vérité de la modernité, à savoir qu'elle n'est jamais changement radical ou révolution, mais qu'elle entre toujours en implication avec la tradition dans un jeu culturel subtil, dans un débat où les deux ont partie liée, dans un processus d'amalgame et d'adaptation. La dialectique de la rupture y cède largement à une dynamique de l'amalgame ${ }^{11}$.

Le processus de modernisation est caractérisé par la transformation des moyens de production (industrialisation) qui passent d'un mode de production agraire et artisanal et d'un système économique basé sur l'autosuffisance à un système de production industriel et à un système d'économie de marché, puis de consommation de masse au $\mathrm{XX}^{\mathrm{e}}$ siècle. Ces transformations sont essentiellement les conséquences d'une révolution des techniques et des technologies, deux révolutions industrielles ${ }^{12}$ qui affectent les sociétés en Occident. Avec l'industrialisation, les sociétés passeront d'un mode de vie rural à un mode de vie principalement urbain. Ces transformations affectent également les représentations du monde et les paradigmes sociaux et politiques.

L'historien Elzéar Lavoie reprend la définition de la notion de modernité de Baudrillard. Lavoie accorde une 
grande importance au processus dynamique de l'amalgame dans son analyse de l'avènement de la modernité populaire au Québec. «Une histoire de la Modernité, nous dit-il, ne peut être qu'une histoire de l'imprimé en langue vulgaire, et de sa lecture par le plus grand nombre ${ }^{13}$.»

Vue en rétrospective, ce qui n'est jamais le cas des contemporains, l'histoire du Québec, depuis la fin du XIX ${ }^{\mathrm{e}}$ siècle, semble celle d'une modernisation inéluctable, en même temps que d'un maintien nécessaire de la tradition. L'imprimé, qui est investi, depuis plus de quatre cents ans, de la fonction de modernisation, rend nécessaire une "politique de la langue », le choix du "vulgaire », du code du plus grand nombre. Les mass médias sont le lieu d'un "curieux compromis » entre modernité et tradition, selon une "dynamique de l'amalgame », au « moment où la société moderne se réfléchit comme telle ", c'est-à-dire au moment où l'idée de démocratie est devenue pratique commune, comme règle du jeu politique, comme résultante de l'alphabétisation généralisée. La tradition, qui est conscience de continuité, s'ajuste en imitant les instruments de la modernité, les absorbe dans son projet de continuité, de telle sorte que la modernisation est toujours à recommencer ${ }^{14}$.

Pour Claude Couture, la presse à grand tirage a été l'un des instruments politiques et idéologiques de la domination des libéraux modérés sur la société québécoise :

Le phénomène idéologique et politique le plus important des années 1867 à 1940 et qui fut négligé par l'historiographie a été l'influence, profonde sur la société québécoise, de ces libéraux modérés et le contrôle du Québec qu'ils ont assuré ou transmis pendant la première moitié du $\mathrm{XX}^{\mathrm{e}}$ siècle $^{15}$. 
Ainsi les médias à grand tirage paraissent être en même temps un signe en soi de la modernité (symbole de l'alphabétisation croissante et de la politisation des masses) et un révélateur, par son contenu, du chassé-croisé modernité-tradition qui animent les sociétés contemporaines. Nous aurons l'occasion de voir si l'analyse du discours des éditorialistes de $\mathrm{La}$ Patrie confirme cette observation générale.

\section{La Patrie et le régime hitlérien}

Cette étude est essentiellement une analyse quantitative et qualitative du contenu de 79 éditoriaux du quotidien La Patrie de 1933 à 1935 et de 1938 à 1939 qui traitaient de l'Allemagne nazie. Les années 1936 et 1937 n'ont pas été retenues car les éditoriaux de La Patrie ne traitaient pas de l'Allemagne durant cette période. D'autres sujets retenaient davantage l'attention des éditorialistes.

Les éditorialistes de La Patrie adoptent une position plutôt défavorable à l'égard de l'Allemagne nazie. On la présente comme étant belliciste et en continuité historique avec les régimes précédents. L'anticatholicisme et le paganisme apparents du nazisme et de son chef incitent les éditorialistes à en offrir une représentation diabolique. Les actions politiques de l'Allemagne à l'étranger qui menacent la paix européenne et l'intensification des crises internationales contribuent à fixer cette image de régime démoniaque. Il faut cependant savoir que la représentation du régime hitlérien n'a pas toujours été aussi négative au cours de la période étudiée. Ainsi, nous avons repéré le moment d'une prise de position claire de la part des éditorialistes. L'année 1938 - et plus précisément le mois de septembre 1938, moment où éclate la Crise des Sudètes représente un point tournant dans le discours des éditorialistes. Deux facteurs peuvent expliquer cette prise de position claire : d'une part, l'intensification des crises politiques inter- 
nationales et, d'autre part, la prise de position du pape Pie XI face au nazisme en 1937. Nous verrons que le discours des éditorialistes reprend alors celui du pape.

Au cours des années précédentes (1933-1935), le ton des éditoriaux est plutôt tiède et modéré. Les éditorialistes critiquaient parfois le régime, mais ils ne le condamnaient pas. Or cette période correspond à l'instauration du régime autoritaire, qui s'est effectuée par la répression, la violence et la brutalité envers les groupes politiques et religieux opposés au régime. Il s'agit également de la période de l'institutionnalisation de l'antisémitisme et du racisme en Allemagne.

Ces aspects du régime hitlérien n'ont que très peu retenu l'attention des éditorialistes qui sont restés muets sur ces questions. Leur intérêt s'est davantage porté sur les questions d'ordre international que sur celles concernant la politique intérieure du régime. Par exemple la promulgation des Lois de Nuremberg ${ }^{16}$, qui constituait un affront à la démocratie libérale, n'a fait l'objet d'aucun commentaire ni d'aucune critique de la part des éditorialistes. On en fait brièvement mention à une occasion mais sans en expliquer les conséquences pour les juifs allemands qui, rappelons-le, perdent alors leur statut de citoyens et leurs droits civiques.

La répression des communistes et des catholiques ne suscitera pas davantage de commentaires. Les éditorialistes semblent beaucoup plus préoccupés par la menace d'un conflit européen, voire mondial. Le régime hitlérien, par ses politiques étrangères et ses projets d'expansion territoriale, est perçu comme l'instigateur potentiel d'un tel conflit. L'intérêt marqué des éditorialistes pour les questions d'ordre international, à la fois dans la première et dans la seconde périodes de notre étude (1933-1935 et 1938-1939), dénote une préoccupation pour la paix internationale. La Crise des Sudètes, en septembre 1938, place les puissances européennes au bord 
du gouffre. La menace d'une guerre devient, à ce moment, réelle. L'accord de Munich qui intervient entre les puissances alliées et l'Allemagne hitlérienne redonnera aux éditorialistes l'espoir que la paix pourra être maintenue.

Au cours de la période 1938-1939, les thèmes de la guerre et de la paix sont omniprésents. Les éditorialistes prennent alors position face au régime hitlérien. Ils caractérisent les forces en présence d'un point de vue moral et dualiste: d'un côté, les forces du Bien, représentées par les puissances démocratiques et libérales (France et Grande-Bretagne); de l'autre, les forces du Mal, représentées par les dictatures fasciste et nazie, païennes et apostates. Les éditorialistes diabolisent alors cet ennemi potentiel :

Qu'y est, en somme, et que représente là-bas [Munich] M. Neville Chamberlain ? Il est le prêtre de la mystique chrétienne. Il incarne le bon sens, l'ordre, la paix sociale. Il plaide la cause de la charité, dans les yeux de l'homme qui affiche la haine en série ou par race. Il revendique les droits de la justice, en face de l'homme qui a voué un culte envers la force armée. Il implore pitié pour une nation à demi immolée, devant l'homme même qui voudrait la déchiqueter toute, avaler les petits peuples. Il s'efforce en un mot de fléchir, d'enchaîner à la paix, une sorte de léopard en liberté, aux yeux de foudre et aux griffes d'acier, de mitraille ${ }^{17}$.

Le régime hitlérien est présenté en continuité historique avec les régimes allemands précédents; on trace un parallèle entre les politiques étrangères du régime hitlérien et les politiques pangermanistes de Bismarck et de Guillaume II. Les éditorialistes utilisent également la référence au passé pour annoncer le destin du régime hitlérien, c'est-à-dire son échec s'il s'engage dans un conflit armé face aux démocraties européennes, comme ce fut le cas pour Guillaume II en 19141918. 
On considère Adolf Hitler comme étant au centre du régime, comme un dictateur fort. Cette perception se confirme après 1938. Pendant la période 1933-1935, le pouvoir d'Adolf Hitler semble, pour les éditorialistes, menacé de l'intérieur par les éléments radicaux du mouvement (SA) et par l'armée ${ }^{18}$. On espère qu'une révolution ou qu'un putsch de l'armée viendra provoquer la chute du Führer. Après 1938, les éditorialistes ne remettent plus en question la stabilité du pouvoir de Hitler. C'est alors le thème de la faiblesse du régime face aux démocraties européennes qui est proposé. Cette supposée faiblesse économique et militaire devient une assurance du maintien de la paix.

L'intensification des crises internationales et la menace imminente d'une guerre consolident la représentation d'un régime diabolique. Le néopaganisme devient alors l'élément justificateur de ce caractère diabolique. Cette dimension de l'idéologie nazie est, pour les éditorialistes, la principale caractéristique du régime hitlérien. Les autres aspects du nazisme sont vus comme des conséquences du caractère païen et barbare de cette idéologie, tout comme les politiques intérieures et extérieures du gouvernement allemand.

Il faut confesser que le nazisme est, au regard du droit et de l'éthique éternelle, une politique assez gênante. Comme son Vicaire de Rome, le Christ l'eût condamné à l'instar du paganisme. Voici. Pour parler franc, le credo hitlérien est aux antipodes du credo apostolique. L'amour du prochain et le respect de la propriété ont en somme peu de logement dans les articles du Symbole nouveau, entre Nuremberg et Nicée, la distance est bien vaste ${ }^{19}$.

Hitler apparait comme un « démon de la force ${ }^{20}$ » qui s'oppose aux démocraties en tant que représentantes de la justice, du droit et de la civilisation. On le compare à «Attila, 
le fléau de Dieu ${ }^{21} »$. On le compare également à Wotan ${ }^{22}$, dieu guerrier de la force dans la mythologie germanique et, par conséquent, dieu païen. Le problème se pose alors dans cet affrontement entre les représentants du Mal et ceux du Bien :

Or, la société se fonde sur la religion du droit. Elle reconnait un Maître et elle en reconnait la doctrine. Sans quoi, elle courrait à l'anarchie et à la déchéance. C'est pour avoir violé cette loi, essentielle à l'ordre et à la durée, que les puissants empires de l'Orient et de l'Occident eurent à crouler, tels des châteaux de cartes. L'enseignement de l'histoire est, là-dessus, lumineux. Or, en pleine clarté, des États qui se donnent des missions, moins élevées encore ou plus fausses que celle d'un Mahomet, prennent le bien d'autrui, renient leur parole après avoir renié le Verbe ou Dieu lui-même, pourrissent le cœur de leurs propres sujets, heurtent effrontément le Décalogue et ses prédicants. [...]

La haine du droit pousse donc à la haine des personnes et des familles ethniques, pourtant égales au regard de Celui qui vient les racheter toutes, chacune ${ }^{23}$.

La persécution des communistes fait exception dans cette représentation somme toute négative du régime hitlérien : c'est le seul moment, après l'accession au pouvoir des nationaux-socialistes en 1933, où le régime hitlérien recevra une certaine approbation. L'anticommunisme des éditorialistes influence alors leur représentation du régime. On approuve les méthodes violentes et répressives employées par le régime afin d'éradiquer le communisme :

Le nouveau chancelier du Reich, Adolph [sic] Hitler, a décidé comme jeu d'entrée, d'en finir immédiatement avec le communisme en lui portant un coup dont il ne se remettra pas de sitôt. Le temps des badinages est passé, semble-t-il dire, et, quels que soient les moyens que nous prenions pour écraser ce monstre, le pays 
s'en trouvera bien. [...] Et, ainsi, les meneurs bolchévistes [sic] qui ont été en Allemagne comme ailleurs la cause de tant de malaises et d'inquiétudes, vont être fauchés et le pays débarrassé de leur poison.

La manière forte vaut mieux que les longues discussions dans bien des cas, et s'il eut fallu que le nouveau chancelier, sous prétexte de respect à la constitution, eût admis que l'on discutât ses intentions, les communistes auraient eu beau jeu ${ }^{24}$.

La question de la répression des communistes n'est vraiment abordée qu'au seul moment de l'incendie du Reichstag dans la nuit du 27 au 28 février $1933^{25}$. Déjà, elle suscite moins d'intérêt dans l'éditorial qui fait suite à l'élection du 5 mars 1933, qui portera le NSDAP (Nationalsozialistische Deutsche Arbeiterpartei: Parti national-socialiste des ouvriers allemands) au pouvoir. Ce thème sera tout à fait absent des éditoriaux de la période 1938-1939. Le fait que les communistes et les socialistes allemands aient été très tôt éliminés de la scène politique peut expliquer ce silence.

L'antisémitisme de même que la répression des juifs et des catholiques occupent également peu de place dans le discours des éditorialistes. Ces aspects semblent peu importants pour les éditorialistes dans la représentation du régime hitlérien. Nous avons dit que le néopaganisme et l'anticatholicisme étaient considérés par les éditorialistes comme les caractéristiques fondamentales de l'idéologie nationale-socialiste. De ce point de vue, la répression des catholiques apparait comme peu surprenante, ce qui explique peut-être pourquoi les éditorialistes ne s'en préoccupent que très peu, d'autant plus que, comme l'un d'eux l'indique à un moment donné, les informations qu'ils recevaient sur la question étaient partielles et incomplètes. Ce mutisme tant sur cette question que sur celle de la répression des juifs et des communistes, est sans doute 
imputable au peu d'intérêt accordé par les éditorialistes aux questions concernant les politiques et les problèmes intérieurs allemands.

Cependant, l'opposition des éditorialistes de La Patrie au nazisme n'est pas que circonstancielle; elle s'appuie sur des fondations idéologiques qui révèlent leur vision du monde.

\section{La vision du monde des éditorialistes de La Patrie}

Le second facteur de la radicalisation du discours de $\mathrm{La}$ Patrie à partir de 1938 serait attribuable à la vision du monde des éditorialistes et aux valeurs qui la sous-tendent. Cette vision du monde, que nous allons ici définir, devient une clé pour la compréhension du processus de modernisation de la société francophone au Québec. Nous proposons ici que le contexte général des idées, comme cadre de référence du discours et de la vision du monde des éditorialistes, est influencé à la fois par des éléments propres à la tradition et par des éléments propres à la modernité ou à une certaine modernité.

Les éditorialistes ont une vision du monde morale et dualiste, fortement empreinte de valeurs chrétiennes et, quoique dans une moindre mesure, de valeurs libérales. C'est cette conception du Bien et du Mal, ancrée dans la morale catholique et les valeurs libérales, qui détermine non seulement leur représentation de l'Allemagne hitlérienne, mais aussi leur conception du communisme et leur position face à cette idéologie au cours de la première période étudiée.

Au cours de la période 1933-1935, les éditorialistes ne portent pas de jugement moral sur le régime hitlérien. Les références aux autorités cléricales et catholiques sont absentes de leur discours. On se réfère davantage aux autorités et aux auteurs laïques et libéraux. Les valeurs libérales que sont la démocratie, la liberté et la propriété privée participent de la 
vision du monde des éditorialistes, qui les considèrent comme des acquis de la civilisation. On retrouvera d'ailleurs des références à ces valeurs tout au long de la période étudiée :

[L'Allemagne] donne avec frénésie dans la psychose de l'autarcie et de l'économie dirigée, du travail conscrit et des taxes à fort voltage. L'homme y est corvéable, plus qu'au moyen âge, avec une marge toute mince de liberté orale ou écrite ${ }^{26}$.

Nos pays démocratiques oublient trop le don insigne de la liberté, d'une liberté décente il va sans dire. Ils oublient également que, sous le régime dictatorial, tout le monde est en tutelle relative, à l'exception des chefs ; que tout le monde y danse, en mouvement commandé ou au pas de l'oie. Et ces chefs dispensent, au comptegouttes, l'information estimée contraire à la mystique officielle et ils filtrent le reste dans le sens d'un conformisme total. [...]

Le procédé totalitaire, qui consiste à traiter les gens comme des pensionnaires collégiaux ou des conventuels, est évidemment opposé aux droits acquis de la civilisation. Il sert l'égoïsme des dirigeants qui réclament sans répit le sacrifice à jet fort, pour la cause, et il veut le moins de lumière possible, même à l'extérieur ${ }^{27}$.

Les valeurs libérales sont intégrées à la conception du Bien chez nos éditorialistes. Or ces principes et ces valeurs doivent être subordonnés aux dogmes supérieurs et aux valeurs du catholicisme. On retrouve donc, dans cette vision du monde, une hiérarchisation des valeurs. Les valeurs spirituelles et morales catholiques trônent au sommet de cette hiérarchie et déterminent les autres valeurs constituant la conception du Bien et du Mal des éditorialistes:

Tôt ou tard $[\ldots]$ les conquérants pressés et les despotes doivent payer le tribut à Celui de qui relève l'univers. La victoire de la force sur le droit n'a qu'un temps ; 
elle appelle, jusqu'à satisfaction, le retour du niveau normal, de l'équilibre. Au-dessus des chefs humains qui contrôlent et détournent à leur guise le bras séculier, il y a le Chef suprême, à la fois Toute-Puissance et Toute-Justice ${ }^{28}$.

L'Europe, en convulsion, cherche la paix. Elle voit, consternée, les hommes se dresser contre les hommes ; l'erreur attenter au vrai éternel; la haine, provoquer l'amour, en combat singulier; une croix païenne, au galbe tourmenté, défier la Croix du Calvaire; la force grossière, fauve, disputer, parfois victorieusement au droit sa royauté $e^{29}$.

Le respect des valeurs spirituelles implique la soumission à l'autorité pontificale. Or le pape ne se prononce officiellement sur la question du national-socialisme qu'en 1937. Pie XI publie, à ce moment, l'encyclique Mit brennender Sorge, dans laquelle il condamne l'idéologie nationale-socialiste mais pas le régime hitlérien. Il publie aussi, la même année, une encyclique condamnant le communisme: Divini Redemptoris. Ainsi, selon l'auteur Marc Agostino:

Pie XI n'envisage pas le national-socialisme comme un danger universel. Il y voit une déviation du nationalisme allemand alors que le communisme lui semble de vocation plus universelle. Cette interprétation restrictive n'empêche pas l'emploi d'un ton très ferme et d'une condamnation sans équivoque du national-socialisme et du racisme ${ }^{30}$.

La position du pape face au régime hitlérien semblait, au cours des années précédentes, équivoque. La signature du Concordat avec le régime en 1933 contribue à cette ambiguïté. À partir de 1937 , le pape condamne cependant le racisme nazi en affirmant qu'il est contraire aux dogmes humanistes du catholicisme. Les éditorialistes adoptent cette position. Ils s'y conforment et s'approprient les propos du pape 
lorsqu'il s'agit de critiquer le régime hitlérien et de prendre position à son égard : «Encore une fois, le Vatican nous enseigne la sérénité ; [...] Le Pape a vigoureusement condamné le racisme ${ }^{31}$.» Pie XI réitérera sa condamnation du nationalsocialisme et du racisme en 1938. Nous pouvons déduire que l'autorité pontificale devient une caution pour les propos des auteurs :

Le récent discours prononcé par le Pape, dans sa résidence d'été de Castelgandolfo, lisons-nous dans l'Agence chrétienne de presse Pax, a marqué une fois de plus et avec une netteté qui ne peut plus tromper personne l'opposition irréductible de la religion catholique, c'est-à-dire universelle, et du racisme allemand tel qu'il est enseigné et appliqué par les chefs du national-socialisme ${ }^{32}$.

Les éditorialistes de La Patrie ont une conception antagoniste de la civilisation et de la barbarie. Cette civilisation, représentée par les États démocratiques et libéraux, a préservé les valeurs et l'humanisme chrétiens. Les États démocratiques qui ont conservé les valeurs spirituelles chrétiennes sont également garants d'une certaine idée du droit. Les éditorialistes font ici référence au droit éternel, donc au droit canon du catholicisme. Le pape est alors l'autorité suprême qui agit au nom du droit et de la volonté divine, ce qui expliquerait ici la prise de position des éditorialistes face au racisme et au régime hitlérien, qui fait suite à celle du souverain pontife :

D'ailleurs, S.S. Pie XI rappelle que « tout le genre humain est une seule et grande race ». Ce qui veut dire que le racisme, soufflé jusqu’à devenir une « religion » politique, est un faux dogme, tout comme le pangermanisme, son application concrète ${ }^{33}$. 
Pie XI s'étant prononcé, les éditorialistes ne peuvent qu'adopter sa position et ses arguments. Leur soumission à l'autorité pontificale, qu'ils réaffirment dans leurs textes, les amène à adopter la position de Rome. C'est là, croyons-nous, la seconde clé à l'explication de la radicalisation du discours des éditorialistes à partir de 1938. La position équivoque de Pie XI à l'égard du régime hitlérien avant 1937 expliquerait vraisemblablement la tiédeur des éditorialistes.

Nous avons souligné le mutisme des éditorialistes sur la question de la répression des catholiques hostiles au régime ainsi que sur celle des juifs et des communistes. Nous ne pouvons affirmer hors de tout doute que les éditorialistes adhéraient aux thèses antisémites. Bien que ces thèses aient été présentes dans la société francophone du Québec à l'époque, nous n'avons repéré, dans le discours des éditorialistes, aucun propos explicitant leur position face à l'antisémitisme, du moins avant 1938. Nous avons repéré, après cette date, des commentaires critiques à l'égard du racisme nazi et de l'antisémitisme. On peut ainsi lire dans un éditorial paru le 6 décembre 1938, soit après la prise de position officielle du souverain pontife, une critique de certaines thèses de l'antisémitisme nazi. L’auteur défend alors les juifs persécutés :

Après avoir subi les pires humiliations et vexations, les Juifs d'Allemagne n'ont cependant pas encore bu leur calice jusqu'à la lie. De nouveaux décrets, plus violents que les précédents, achèvent de leur enlever le peu qu'on leur avait laissé. [...]

Après avoir accusé les Juifs d'avoir été les instigateurs de la guerre mondiale, on leur reprocha d'avoir causé la défaite des troupes du Kaiser. Mais il fallait trouver plus tard d'autres prétextes pour « justifier » cette guerre qui se livre sans armes. Les Juifs furent alors accusés de ruiner économiquement la nouvelle Allemagne, de fomenter la révolution et l'anarchie. 
Numériquement, les Juifs constituent à peine un pour cent de la population mondiale. Ce un pour cent est dispersé dans tous les pays du monde. Politiquement, ils n'ont aucune importance, étant donné leur incapacité de s'organiser en tant que peuple. C'est là la preuve qu'ils ne peuvent, ainsi qu'on le prétend, dominer financièrement ou industriellement. Individuellement, ils ont toujours exercé une forte influence sur le monde des arts et des sciences, car, le Juif est foncièrement intellectuel. Sa contribution a toujours été considéra$\mathrm{ble}^{34}$.

Le régime hitlérien est l'antithèse de la société idéale des éditorialistes. Ce régime est païen et barbare, il a rejeté Dieu et les dogmes catholiques. La violence, l'autoritarisme, la soif de puissance et de conquête, l'esprit belliciste, la négation des libertés et de la propriété privée seraient des conséquences de ce paganisme, de ce rejet des dogmes et des valeurs spirituelles catholiques.

La vision du monde des éditorialistes est donc influencée par les valeurs traditionnelles : la foi, la soumission aux dogmes et à l'autorité catholiques, le maintien et le respect des hiérarchies sociales, la foi dans un monde déterminé par la volonté divine et par la Providence. Cette influence de valeurs associées à la tradition ne nous permet pas de conclure pour autant que le discours des éditorialistes n'a rien de moderne. On y remarque également l'influence d'idées associées au libéralisme et à une certaine conception de la modernité : une conception du politique, de l'État, de la légitimité démocratique de l'État libéral - légitimité qui ne provient pas de Dieu mais de la volonté du peuple - les valeurs libérales de liberté et de propriété privée:

[...] le pape s'est élevé avec force contre la philosophie d'une politique envahissante qui vise à la division de l'humanité, à l'anéantissement de la liberté individuelle, 
dans un but évident d'hégémonie au profit de quelques dictateurs ${ }^{35}$.

La vision du monde des éditorialistes est également influencée par des idées nouvelles dans les années 1930, mais qui sont, avec le recul du temps, associées au conservatisme et au traditionalisme :

La personne humaine étouffe dans un univers qui ne cherche qu'à satisfaire ses ambitions collectives. Et si on l'examine à la loupe, on constate que le problème moderne se résume à cette alternative : Rétablir l'ordre moral des valeurs ou sombrer dans le cataclysme que nous prépare le matérialisme, dans l'ambiance duquel nous vivons. [...] mais la seule révolution qui apporterait un changement radical à l'état de chose actuel — la révolution spiritualiste - personne n'en parle. [...]

Il ne suffit plus, aujourd'hui, de diviser les hommes en fascistes, communistes et démocrates ; la crise actuelle ne peut avoir d'autre issue qu'une issue spiritualiste ou une issue matérialiste. [...] Et le Catholicisme peut remodeler le monde si les catholiques veulent se persuader de l'urgence qu'il y a à adopter une attitude vivante et dynamique, une attitude qui soit combative et conquérante ${ }^{36}$.

Le projet de révolution spirituelle proposé ici par l'éditorialiste peut nous apparaittre aujourd'hui comme une idée conservatrice et réactionnaire au progrès porté par le libéralisme et la rationalité. Ce projet est présenté comme étant la voie idéale du progrès de l'humanité. De ce fait, il légitime la critique du monde contemporain. Le conservatisme apparent des éditorialistes ne s'opposerait donc pas nécessairement à la modernité. Pourrions-nous définir cette idéologie, à la suite de Fernande Roy ${ }^{37}$ et de Claude Couture ${ }^{38}$, comme un libéralisme classique et archaïque qui aurait plutôt bien cohabité 
avec les valeurs catholiques et traditionnelles dans la première moitié du $\mathrm{XX}^{\mathrm{e}}$ siècle?

\section{$* * *$}

Nous avons vu que deux facteurs ont influencé la prise de position des éditorialistes de La Patrie dans leur représentation du régime hitlérien après 1938. Le premier est lié à leur crainte d'un nouveau conflit mondial, qui se fait plus menaçant à partir de 1938 avec l'intensification des crises internationales, notamment avec la Crise des Sudètes. Le second facteur est lié à la prise de position du pape Pie XI en 1937 sur la question du national-socialisme allemand. Celui-ci nous parait révélateur de la vision du monde des éditorialistes. Il est déterminant dans la représentation du régime hitlérien par les éditorialistes du quotidien La Patrie.

Nous voulions, par notre analyse, mettre la vision du monde des éditorialistes en rapport avec les connaissances du contexte général des idées au Québec dans les années 1930. Ce contexte était alors caractérisé par la mutation de la société québécoise francophone. Dans un contexte de modernisation qui prend naissance à la fin du XIX $\mathrm{X}^{e}$ siècle, les valeurs traditionnelles sont confrontées à de nouvelles valeurs.

Le processus de modernisation est un processus dynamique d'amalgame de tradition et de modernité. Il a été possible de repérer dans le discours des éditorialistes cette coexistence de valeurs traditionnelles et libérales. La vision du monde des éditorialistes de La Patrie participe de ce processus de modernisation de la société québécoise francophone. Les valeurs traditionnelles sont encore influentes dans les années 1930, mais ces valeurs ont été adaptées par l'intégration de valeurs libérales et de traits caractéristiques de la modernité à une conception morale de la civilisation. La radicalisation du discours et de la position des éditorialistes face au régime hit- 
lérien, qui fait suite à la prise de position officielle du pape Pie XI, témoigne néanmoins d'une soumission à l'autorité pontificale et, par conséquent, de l'influence prépondérante des valeurs et du dogme catholiques véhiculés par les discours et les encycliques du pape.

Cette analyse nous a permis d'exposer la vision du monde des éditorialistes au cours d'une période précise de l'histoire du Québec contemporain. Nous savons, avec le recul, que la vision morale et plutôt conservatrice du monde des éditorialistes, si elle semble dominante dans les années 1930, sera supplantée, trente ans plus tard, par une autre vision du monde, libérale celle-là. Les éditorialistes de La Patrie ne remettent pas en question ces valeurs du libéralisme, mais, comme nous l'avons vu, ils les intègrent à une conception morale du monde. Par contre, cette analyse partielle, limitée aux éditoriaux de La Patrie, ne peut rendre compte adéquatement du processus de modernisation de la société francophone québécoise. Pour ce faire, il nous aurait fallu comparer ce discours avec celui des époques précédant et suivant la période étudiée. Le processus de modernisation de la société québécoise s'effectue sur le long terme. Il prend naissance à la fin du XIX ${ }^{\mathrm{e}}$ siècle avec les transformations que provoquent l'industrialisation et l'urbanisation et culmine dans les années 1960-1970. Notre analyse, qui porte sur une courte période (1933-1939), n'est que le portrait d'un moment de ce processus.

\section{NOTES}

' Jacques Pelletier, «La Relève: une idéologie des années 1930 », Voix et images dupays, $\mathrm{n}^{\circ} \mathrm{V}, 1972$, p. 83.

${ }^{2}$ Alfred Grosser. Hitler, la presse et la naissance d'une dictature. Paris, Armand Collin, 1972. 215 p. 
${ }^{3}$ Guy Massicotte. «Les éditorialistes canadiens-français et les origines de la Seconde Guerre mondiale ». Recherches sociographiques, vol. 17, $\mathrm{n}^{\circ}$ 2, (mai-août 1976), pp. 139-165.

+ Françoise Caron-Houle. La presse française du Québec et les crises européennes, 1935-1939. Thèse de maitrise, Université d'Ottawa, 1972. 202 p.

${ }^{5}$ Claude Couture, Le mythe de la modernisation du Québec. Des années 1930 à la révolution tranquille, Montréal, Éditions du Méridien, 1991, p. 122.

"André Beaulieu, Jean Hamelin, et. al, La presse québécoise des origines à nos jours. Tome troisième, Sainte-Foy, Les Presses de l'Université Laval, 1977, pp. 287-290.

${ }^{7}$ Luc Côté et Jean-Guy Daigle, Publicité de masse et masse publicitaire. Le marché québécois des années 1920 aux années 1960, Ottawa, Les Presses de l'Université d'Ottawa, 1999, p. 45.

${ }^{8}$ Marcel Fournier. L'entrée dans la modernité. Science, culture et société au Québec. Montréal, Éditions Saint-Martin, 1986. 239 p.

"Jean Baudrillard, «Modernité », Encyclopadia Universalis, vol. 15, Paris, 1990, p. 552.

${ }^{10}$ Ibid., p. 552.

${ }^{11}$ Ibid., p. 553.

${ }^{12}$ François Caron. Les deux révolutions industrielles du $X X^{c}$ siècle. Paris, Albin Michel, 1997. $525 \mathrm{p}$.

${ }^{13}$ Elzéar Lavoie, «La constitution d'une modernité culturelle populaire dans les médias au Québec (1900-1950) ", dans Yvan Lamonde et Esther Trépanier, dir. L'avènement de la modernité culturelle au Québec, Québec, IQRC, 1986, p. 255.

${ }^{1+}$ Loc. cit., p. 287.

${ }^{15}$ Claude Couture, op. cit, p. 31.

${ }^{16}$ Le 15 septembre 1935, le gouvernement nazi promulgue les Lois de $\mathrm{Nu}$ remberg qui ont pour effet de retirer les droits de citoyenneté aux juifs allemands et d'interdire les relations sexuelles et les mariages interraciaux. Il semble que cet événement n'ait pas retenu l'attention des éditorialistes car nous n'avons trouvé aucun éditorial portant spécifiquement sur lui. Or, nous savons que les éditorialistes étaient au fait des événements car, d'une part, nous avons retrouvé un entrefilet qui mentionnait ces décrets, et, d'autre part, l'éditorialiste fait référence à une occasion à ces décrets dans un texte. 
${ }^{17}$ Léon Gray, «Face au Rhin. Deux mystiques », La Patrie, 23 septembre 1938, p. 10.

${ }^{18}$ Les SA sont neutralisés comme force politique lors de la Nuit des longs couteaux, le 30 juin 1934. Leur chef Ernst Röhm et ses principaux collaborateurs sont alors assassinés. Le régime hitlérien met fin aux projets de fusion de la Reichswher (armée allemande) et des SA qui auraient pu menacer le pouvoir nazi.

${ }^{19}$ Léon Gray, «La furie prussienne. Eclose en Autriche », La Patrie, 14 octobre 1938, p. 10.

201 Idem, «La paralysie mondiale. Point névralgique », La Patrie, $1^{\text {er }}$ septembre 1938 , p.10

${ }^{21}$ Idem, «L'esprit de Munich. A Londres et Paris », La Patrie, 15 novembre 1938 , p. 24.

${ }^{22}$ Idem, «Le sage de Nuremberg. Adolf Hitler», La Patrie, 7 septembre 1938, p. 10.

${ }^{23}$ Idem, « Rétrospective. L'année moribonde », La Patrie, 30 décembre 1938, p. 10.

24 ..---, «Le bras de fer d'Hitler », La Patrie, $1^{\text {er }}$ mars 1933, p. 4.

${ }^{25}$ Cet incendie devient un prétexte pour arrêter massivement des militants communistes et socialistes. Ces deux partis seront interdits par décrets.

${ }^{26}$ Léon Gray, «Le drame de la paix. Chamberlain repart », La Patrie, 22 septembre 1938, p. 10.

${ }^{27}$ Idem, « M. Chamberlain. Il a parlé fort », La Patrie, 14 décembre 1938, p. 10.

${ }^{28}$ Idem, « La crise européenne. Ses causes et leçons », L a Patrie, 31 août 1938, p. 10.

${ }^{29}$ Idem, "De l'Europe volcanique. Au No. 520, Driveway ", La Patrie, 24 septembre 1938, p. 24.

${ }^{30}$ Marc Agostino, Le pape Pie XI et l'opinion (1922-1939), Rome, École française de Rome, 1991, p. 640.

31 -----, « La paix. Il faut l'instaurer», La Patrie, 20 septembre 1938, p. 10.

32 ------, « Poison de l'humanité. Le racisme », La Patrie, 29 août 1938, p. 10.

33 -.----, «La paix. Il faut l'instaurer », La Patrie, 20 septembre 1938, p. 10. 
${ }^{34}$ Hervé de Saint-Georges, «La persécution des Juifs. Rêve d'hystérique », La Patrie, 6 décembre 1938, p. 10.

35 -.-.- , « Poison de l'humanité. Le racisme », La Patrie, 29 août 1938, p. 10.

${ }^{36}$ Dostaler O'Leary, "Spiritualisme et matérialisme. Problème actuel », La Patrie, 13 mars 1939, p. 10.

${ }^{37}$ Fernande Roy. Histoire des idéologies au Québec aux XIX et XX $X^{e}$ siècles. Montréal, Boréal, 1993. 127 p.

${ }^{38}$ Claude Couture, op. cit., pp. 122-123. 